\title{
SAVR and TAVR: The Role of the Heart Team Towards a Tailored Approach. Benoit de Varennes MD, MSc, FRCS
}

\author{
Benoit de Varennes ${ }^{1}$ \\ ${ }^{1}$ McGill University
}

September 14, 2020

\begin{abstract}
The well-accepted role of the Heart Team in assessing patients suffering from aortic stenosis is becoming the standard approach in most centers. A tailored approach to individual patients may lead to significant changes in outcomes even though SAVR will continue to play a major rollin the treatment of patients presenting more co-morbidities and anatomical challenges.
\end{abstract}

\section{SAVR and TAVR: The Role of the Heart Team Towards a Tailored Approach.}

Benoit de Varennes MDCM, MSC, FRCS

McGill University Health Centre

Division of Cardiac Surgery

1001 Boul. Décarie

Montréal, QC, Canada H4A 2M3

benoit.devarennes@muhc.mcgill.ca

In this issue of the Journal (1), a group from the Université de Montréal, Canada, describes a retrospective analysis of 812 consecutive intermediate-risk patients who were treated for isolated aortic stenosis between 2012 and 2019. All patients who underwent trans-catheter aortic valve replacement (TAVR) were rigorously reviewed by a Heart Team and bioprosthetic surgical aortic valve replacement (SAVR) patients done during the same period were included. The authors do not mention on which basis surgical patients received the treatment.

Applying propensity matching, a total of 139 patients were retained in each group. The trans-femoral approach for TAVR was used in $86 \%$ of patients with the Edwards Sapien platform used in the majority of patients. 99\% of surgical patients were done through a full median sternotomy using almost exclusively the Edwards Magna or Perimount prostheses.

As expected, the types of complications greatly differed between the 2 groups: TAVR was associated with higher rates of transient ischemic attacks, need for permanent pacemaker and para-valvular leak. SAVR was associated with increased rates of acute kidney injury, atrial fibrillation, delirium, infections, bleeding complications and increased length of stay. The STS predicted 30-day mortality was similar between the 2 groups (4,5\% for SAVR, 4,8\% for TAVR). The observed mortality was not significantly different for the 2 cohorts (SAVR $=4,5 \%$ TAVR $=0,7 \% p=0,053$ ) in the matched analysis. Such results have already been described in many trials, the most well-known being Partner 2A (2).

What makes this paper interesting is the angle the authors have given to their data. The evolution of the trend from SAVR to TAVR between 2012 and 2019 in this academic center is not mentioned in the paper. 
The matched analysis indicates that there were similar numbers of patients in each cohort for each 2-year block, but we can safely assume that over that period, the population of SAVR vs TAVR has significantly changed like in all other busy centers. It is likely that ALL patients (or the majority) requiring treatment for aortic stenosis are now evaluated by a Heart Team as opposed to the beginning of their series. This usually causes a paradigm shift in which aortic stenosis patients who are NOT candidates for TAVR (root anatomy, sub-annular calcifications, etc.) end up in the surgical arm. These patients usually carry quite an elevated risk of mortality and complications which are not taken into account with the current risk-prediction models (STS score, EURO score) we use nowadays and may explain the trend towards a higher mortality. As more and more of these patients will receive SAVR, a significant advantage (as opposed to the current non-significant difference) will be seen as an index of superiority favoring TAVR. This is where the Heart Team approach is crucial as it is the only entity that can determine what the best approach will be for individual patients. With this tailored approach, cardiologists and surgeons will have to play a major role in educating patients and referring physicians. The days of only quoting literature results are over unless newer scoring systems are developed and taking into consideration the anatomical limitations for each approach.

The authors discuss the role of blood transfusions during SAVR leading to higher rates of any morbidity/mortality. Minimal-access SAVR and rapid-deployment SAVR being associated with lesser bleeding, shorter length of stay and more rapid extubation $(3,4)$ might mitigate the results of this study as only $1 \%$ of the SAVR patients were done through a minimal-access approach. Using those newer techniques might potentially narrow the gap between TAVR and SAVR.

While the mortality trends for SAVR have been quite stable over the last decade, the authors have demonstrated a clear reduction in the observed mortality of patients being treated for aortic stenosis (TAVR and SAVR combined) in this experienced academic center between 2019 and 2019. This can only be explained by the role of the Heart Team in tailoring the approach to such patients.

The authors should be commended for their work.

Benoit de Varennes

MDCM, MSc, FRCS

McGill University Health Centre

\section{References}

1. KN Khalil, M Boukhris, M Badreddine, W Ben Ali, L-M Stevens, J-B Masson, J Potvin, J-F Gobeil, N Noiseux, P Khairy, J Forcillo. Changes in Outcomes Over Time in Intermediate-Risk Patients Treated for Severe Aortic Stenosis. J Card Surg...............

2. Smith CR, Leon MB, Mack MJ, Miller DC, Moses JW, Svensson LG, Tuzcu EM, Webb JG, FontanaGP, Makkar RR, Williams M, Dewey T, Kapadia S, Babaliaros V, Thourani VH, Corso P, Pichard AD,Bavaria JE, Herrmann HC, Akin JJ, Anderson WN, Wang D, Pocock SJ and Investigators PT.Transcatheter versus surgical aortic-valve replacement in high-risk patients. N Engl JMed.2011;364:2187-98.

3. ML Brown, SH McKellar, TM Sundt, HV Schaff. Ministernotomy versus conventional sternotomy for aortic valve replacement: A systematic review and meta-analysis. J Thorac Cardiovasc Surg 2009; 137: $670-9$.

4. M Glauber, A Micelli, D Gimanov, M Ferrarini, S Bevilacqua, PA Farneti, M Solinas. Right anterior minithoracotomy versus conventional aortic valve replacement: a propensity score matched study. J Thorac Cardiovasc Surg 2013; 145: 122-6. 